\title{
Manipulation and transport of antiprotons for an efficient production of antihydrogen atoms
}

\author{
Minori Tajima ${ }^{1,2}$, Naofumi Kuroda ${ }^{1}$, Pierre Dupre ${ }^{2}$, Yugo Nagata ${ }^{2,3}$, Balint Radics ${ }^{2}$, \\ Takuya Matsudate ${ }^{1}$, Marco Leali ${ }^{4}$, Valerio Mascagna ${ }^{4}$, Luca Venturelli ${ }^{4}$, \\ Horst BreukeR $^{5}$, Hiroyuki Higaki ${ }^{6}$, Yasuyuki $\mathrm{KANAI}^{2}$, Evandro Lodi RizzinI ${ }^{4}$, \\ Yasuyuki Matsuda ${ }^{1}$, Stefan Ulmer $^{7}$ and Yasunori YamaZAKI ${ }^{2}$ \\ ${ }^{1}$ The University of Tokyo, 3-8-1 Komaba, Meguro, Tokyo 153-8902, Japan \\ ${ }^{2}$ Atomic Physics Research Unit, RIKEN, 2-1 Hirosawa, Wako, Saitama 351-0198, Japan \\ ${ }^{3}$ Department of Applied Physics, Tokyo University of Agriculture and Technology, Koganei, Tokyo \\ 184-8588, Japan \\ ${ }^{4}$ University of Brescia, 15 Piazza del Mercato, Brescia BS 25121, Italy \\ ${ }^{5}$ CERN, 23 Geneva, CH 1211, Switzerland \\ ${ }^{6}$ Hiroshima University, 1-2-3 Kagamiyama, Higashihiroshima, Hiroshima 739-8530, Japan \\ ${ }^{7}$ Ulmer Initiative Research Unit, RIKEN, 2-1 Hirosawa, Wako, Saitama 351-0198, Japan \\ E-mail: tajima@radphys4.c.u-tokyo.ac.jp
}

(Received June 15, 2016)

The ASACUSA collaboration is developing methods to extract and transport antiprotons at $20 \mathrm{eV}$ adiabatically for an efficient production of cold antihydrogen atoms using a direct injection scheme. We successfully observed a high production rate of antihydrogen atoms immediately after the mixing of antiprotons.

KEYWORDS: antiproton, antihydrogen

\section{Introduction}

The ASACUSA collaboration has been making efforts to test CPT symmetry by comparing spectroscopic properties between hydrogen and antihydrogen atoms. Our goal is a measurement of the hyperfine transition frequency of ground-state antihydrogen atoms. Figure 1(a) shows a schematic view of our setup renewed in 2014. There are an antiproton trap called the MUSASHI trap consisting of a superconducting solenoid and multi-ring electrodes (MRE) [1], a positron accumulator, a double cusp trap consisting of a two pairs of superconducting anti-Helmholtz coils [2] and MRE, and a spectroscopy line consisting of a microwave (MW) cavity, a sextupole magnet and an antihydrogen detector. Antihydrogen atoms are produced in the double cusp trap. Then a polarized beam in low field seeking states is extracted to the downstream side because of a magnetic field gradient of the double cusp magnet. When an antihydrogen atom is on resonance at the MW cavity, the antihydrogen atoms in low field seeking states are converted into high field seeking states, which are defocused by the sextupole magnet. On the other hand, when an atom is off resonance, the atomic beam polarized in low field seeking states is focused by the sextupole magnet onto the antihydrogen detector. Therefore the transition frequency is determined from a dependency of a count rate of antihydrogen detector as a function of the frequency of the microwaves applied to the cavity. 
(a)

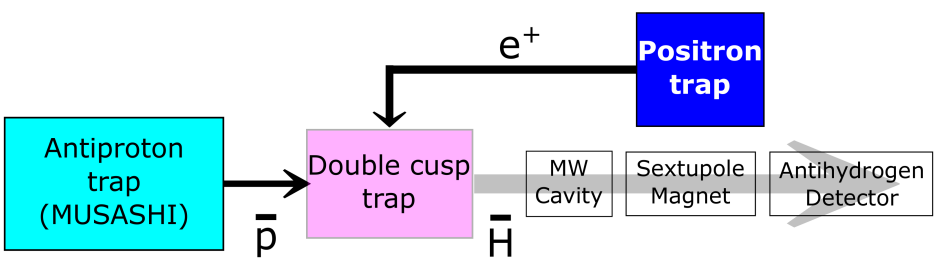

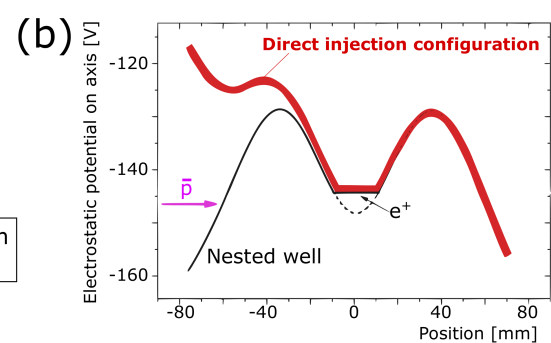

Fig. 1. (a) A schematic diagram of the experimental setup. (b) An electrostatic potential configuration on axis of the mixing region in the cusp trap.

\subsection{The way to produce antihydrogen atoms}

Antiprotons are supplied from the Antiproton decelerator of CERN. They are accumulated, cooled by pre-loaded electrons, and radially compressed in the MUSASHI trap. Then the cooled antiprotons are injected into positrons pre-loaded in the cusp trap to produce antihydrogen atoms. Figure 1(b) shows an electrostatic potential on axis at the cusp trap. Positrons are confined in the bottom of the central potential well which is called nested well. When the antiprotons are injected into the double cusp trap, the potential configuration is changed to the one shown in thick line for a few tens of microseconds, then the potential is switched back to the nested well in order to trap antiprotons. We call this mixing scheme direct injection.

\subsection{Requirements for a spectroscopy}

As many antihydrogen atoms as possible are needed for a high precision measurement to get higher statistics. In addition, cold antihydrogen atoms are necessary in order to extract a polarized beam because the sextupole magnet is designed for an antihydrogen beam of $100 \mathrm{~K}$ or lower. In principle, the velocity of an antihydrogen atom is determined by the velocity of injected antiprotons. Regarding positrons, they are cooled down by cyclotron radiation in the order of second during confinement in the double cusp trap before injection of antiprotons (the magnetic field strength is about $2 \mathrm{~T}$ in the positron trapping region). For antiprotons, the injection energy with respect to the potential of the positron plasma can be changed by simply floating the MUSASHI trap as a whole. Therefore the velocity of antiprotons in the double cusp trap determines the velocity of antihydrogen atoms.

\section{$1.3 \bar{H}$ beam during beamtime in 2012}

During beamtime in 2012, we used a single cusp magnet, antiprotons were injected at $150 \mathrm{eV}$ into positrons, and we succeeded in detecting antihydrogen atoms at $2.7 \mathrm{~m}$ downstream of the mixing region [3]. But a production rate of antihydrogen atoms reaches its maximum about $20 \mathrm{~s}$ after the mixing. Figure 2(a) shows an axial energy spread of antiprotons at the entrance of the mixing region. Figure 2(b) shows the way to measure the energy spread. A potential shown in blue solid line was prepared and changed to the one shown in dotted line at the injection of antiprotons in order to capture them. Those which have lower axial energy than the barrier were reflected and those which have higher axial energy than the barrier were trapped. Although the energy spread of antiprotons in the MUSASHI trap is estimated at sub eV [4], the axial energy spread was estimated at about $20 \mathrm{eV}$, which was unexpectedly large. It is explained by gaining radial energy depending on each trajectory at the entrance of the cusp trap where the magnetic field becomes strong again after a transport line where the magnetic field is weak. Because of this large axial energy spread of antiprotons, positrons are heated up and it takes time to be cooled down in order to form an antihydrogen atom (Fig. 3(a)). One solution to avoid unnecessary heating of positrons is to decrease the injection energy of antiprotons with respect to the positrons and use only a part of antiprotons for the production of 
antihydrogen atoms (Fig. 3(b)), at the cost of antihydrogen number.

(a)

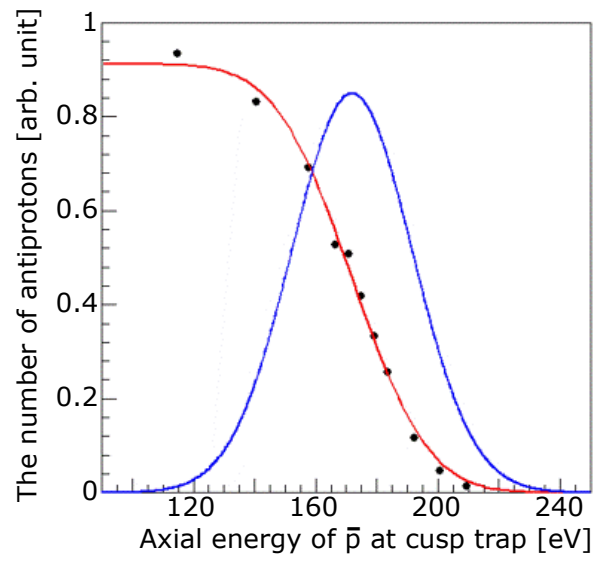

(b)

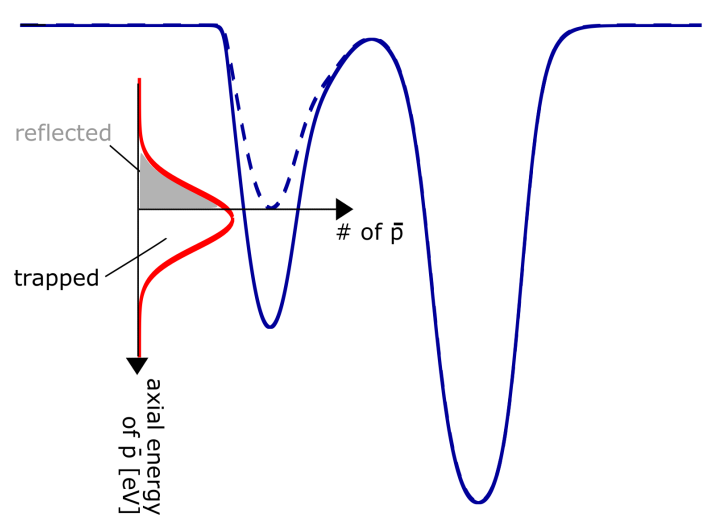

Fig. 2. (a) An axial energy spread of antiprotons measured at the entrance of the cusp trap during beamtime in 2012. The plot shows the number of trapped antiprotons as a function of the height of the barrier at the entrance. Red solid line shows a integral of a gaussian distribution shown by a blue solid line. (b) A scheme used for the measurement of the axial energy spread of antiprotons.
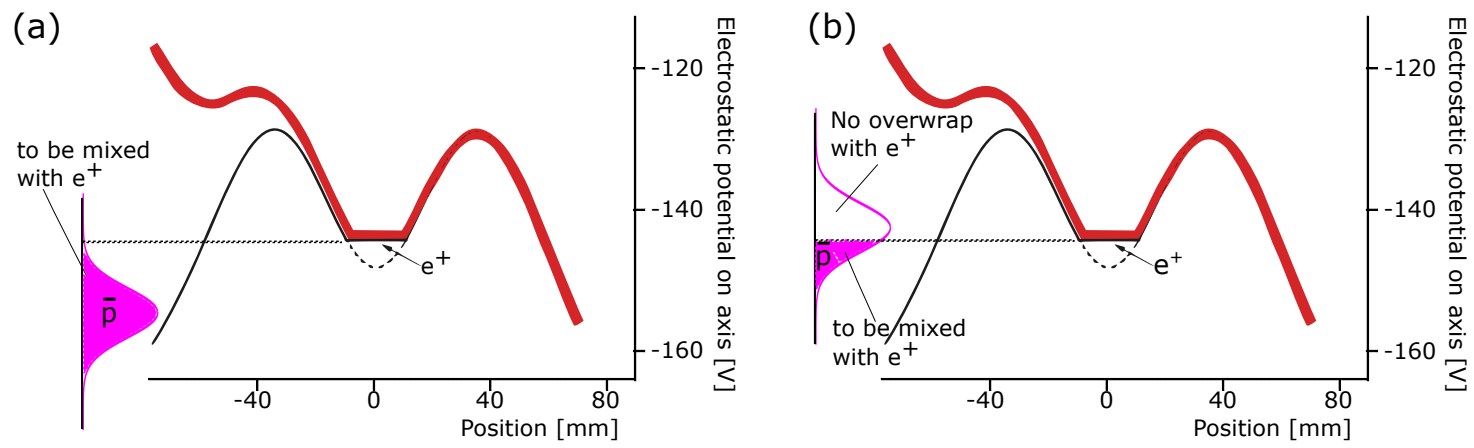

Fig. 3. (a) A schematic diagram of a direct injection method with a broad axial energy spread of antiprotons. Filled gaussian distribution represents an axial energy distribution of antiprotons. (b) A schematic diagram with a broad axial energy spread of antiprotons when the injection energy of antiprotons is changed. 


\section{Manipulation and transport of $\bar{p}$ for an efficient production of $\bar{H}$ atoms}

As described in the previous section, the axial energy spread of antiprotons became large in the cusp trap during beamtime in 2012. In this section, some transport features are discussed employing calculated trajectories. Then extraction schemes from the MUSASHI trap are discussed trying to reduce the energy spread.

\subsection{Transport of $\bar{p}$}

Figure 4(a) shows calculated trajectories of antiprotons with its initial radial position of $0.4,0.8$, 1.2 , and $1.6 \mathrm{~mm}$ in the MUSASHI trap together with magnetic field lines. In this calculation, our setup including the solenoid of the MUSASHI trap, electrostatic lens, DC transport coils, and the double cusp magnet are considered. Their axial positions along the beamline are indicated by the colored area. The injection energy of antiprotons is $150 \mathrm{eV}$. Trajectories are bent at electrostatic lens and antiprotons do not follow the magnetic field line, which means transport is far from being adiabatic. This non-adiabatic transport is the first reason of the large axial energy spread of antiprotons in the cusp trap. Therefore it is important to realize a transport of antiprotons following the magnetic field lines more adiabatically.

At $150 \mathrm{eV}$, the transport of antiprotons is not adiabatic with the magnetic fields shown in Fig. 4(a). In order to realize an adiabatic transport, the injection energy of antiprotons is lowered down to $20 \mathrm{eV}$, and also the magnetic field at the exit of the MUSASHI trap is increased. Figure 4(b) shows trajectories of antiprotons at $20 \mathrm{eV}$ with the new magnetic field configuration. The trajectory simulation results indicate that the trajectories are more adiabatic.

(a)

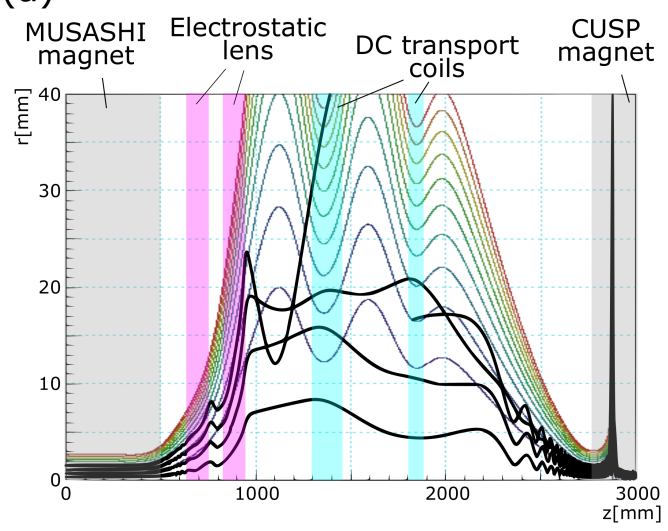

(b)

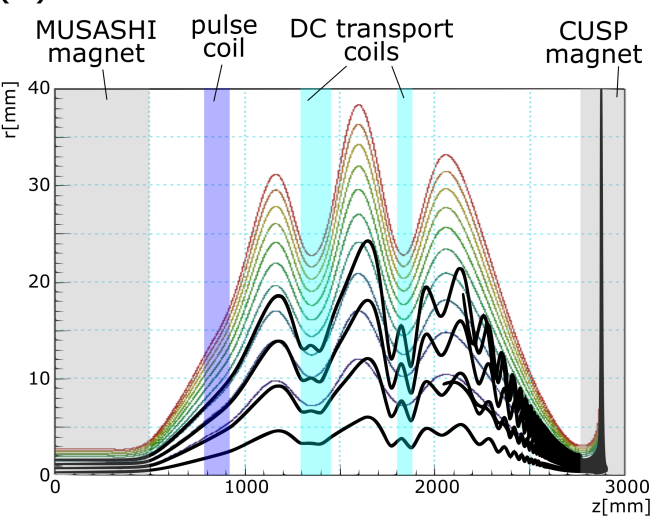

Fig. 4. Calculated trajectories of antiprotons and magnetic field lines (a) for injection energy at $150 \mathrm{eV}$ using electrostatic lens and DC transport coils with its current of $15 \mathrm{~A}$, (b) for injection energy at $20 \mathrm{eV}$ using transport coils with its increased current of $50 \mathrm{~A}$ and a new pulse coil at the exit of the MUSASHI trap.

\subsection{Extraction of $\bar{p}$}

The energy spread of extracted antiprotons from the MUSASHI trap is also important for the production of antihydrogen atoms. To measure the energy spread of antiprotons, annihilation time spectra at the positions of $1.5 \mathrm{~m}$ and $2.1 \mathrm{~m}$ from the MUSASHI trap were observed. Figure 5(a) shows such annihilation spectra when antiprotons are extracted by changing the potential from the solid line to the dotted line in Fig. 5(b). The spectrum is normalized by its area. When the antiprotons are stopped at $2.1 \mathrm{~m}$, the spectrum is broader than that at $1.5 \mathrm{~m}$, indicating the energy spread is 
considerable. Figure 5(c) shows annihilation spectra when antiprotons are extracted as shown in Fig. $5(\mathrm{~d})$. When the antiprotons are stopped at $2.1 \mathrm{~m}$, the spectrum is almost the same as the spectrum at $1.5 \mathrm{~m}$, indicating the energy spread becomes smaller.

(a)

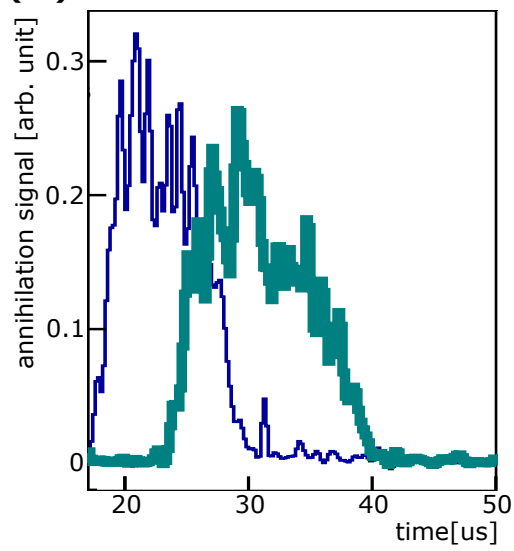

(c)

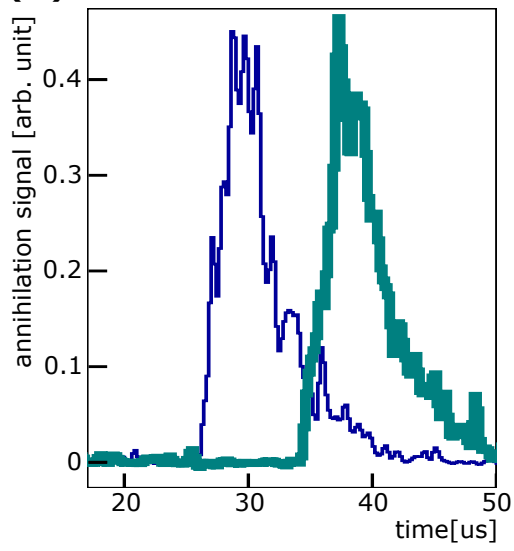

(b)

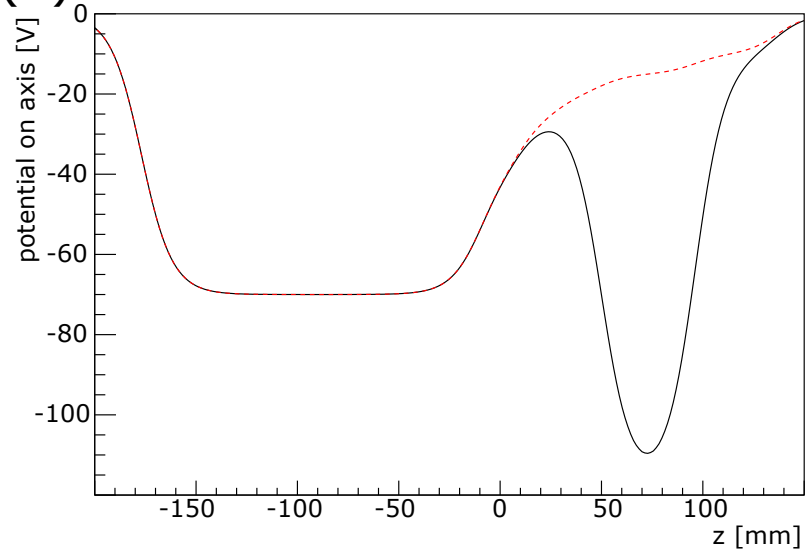

(d)

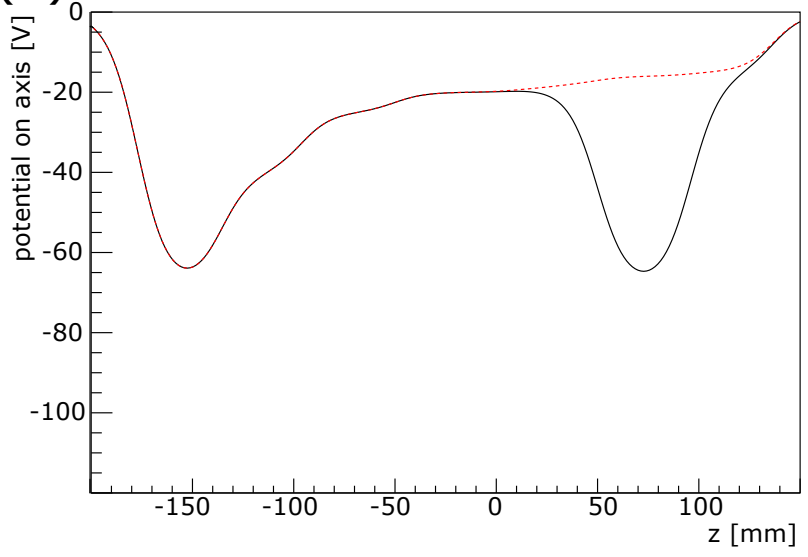

Fig. 5. (a) Annihilation spectra at $1.5 \mathrm{~m}$ and $2.1 \mathrm{~m}$ from the center of the MUSASHI trap when antiprotons are extracted as shown in (b). (b) A potential configuration at extraction from the MUSASHI trap, trying to shorten a bunch length of antiprotons for higher catching efficiency at the double cusp trap. (c) Annihilation spectra when antiprotons are extracted as shown in (d). (d) Another potential configuration at extraction from the MUSASHI trap, trying to keep the potential difference in the region of trapped antiprotons small between the solid line and the dotted line.

\subsection{Production rate of $\bar{H}$ atoms}

Figure 6 shows time dependences of antihydrogen production. The thick magenta line shows the result when the extraction scheme of antiprotons shown in Fig. 5(d) was used and antiprotons were transported as shown in Fig. 4(b). The number of antihydrogen events was 158 for first 0.5 $\mathrm{s}$ in 2015 compared to 6 for first $5 \mathrm{~s}$ in 2012. Antihydrogen atoms were produced at higher rate immediately after the mixing in 2015 , without a delay of 20 s for cooling of positrons which had been heated by injected antiprotons as observed in 2012. It implies a smaller relative energy of antiprotons with respect to the potential of the positron plasma. Since the velocity of antiprotons determines the velocity of antihydrogen atoms as described in section 1.2, it also implies colder antihydrogen atoms. 


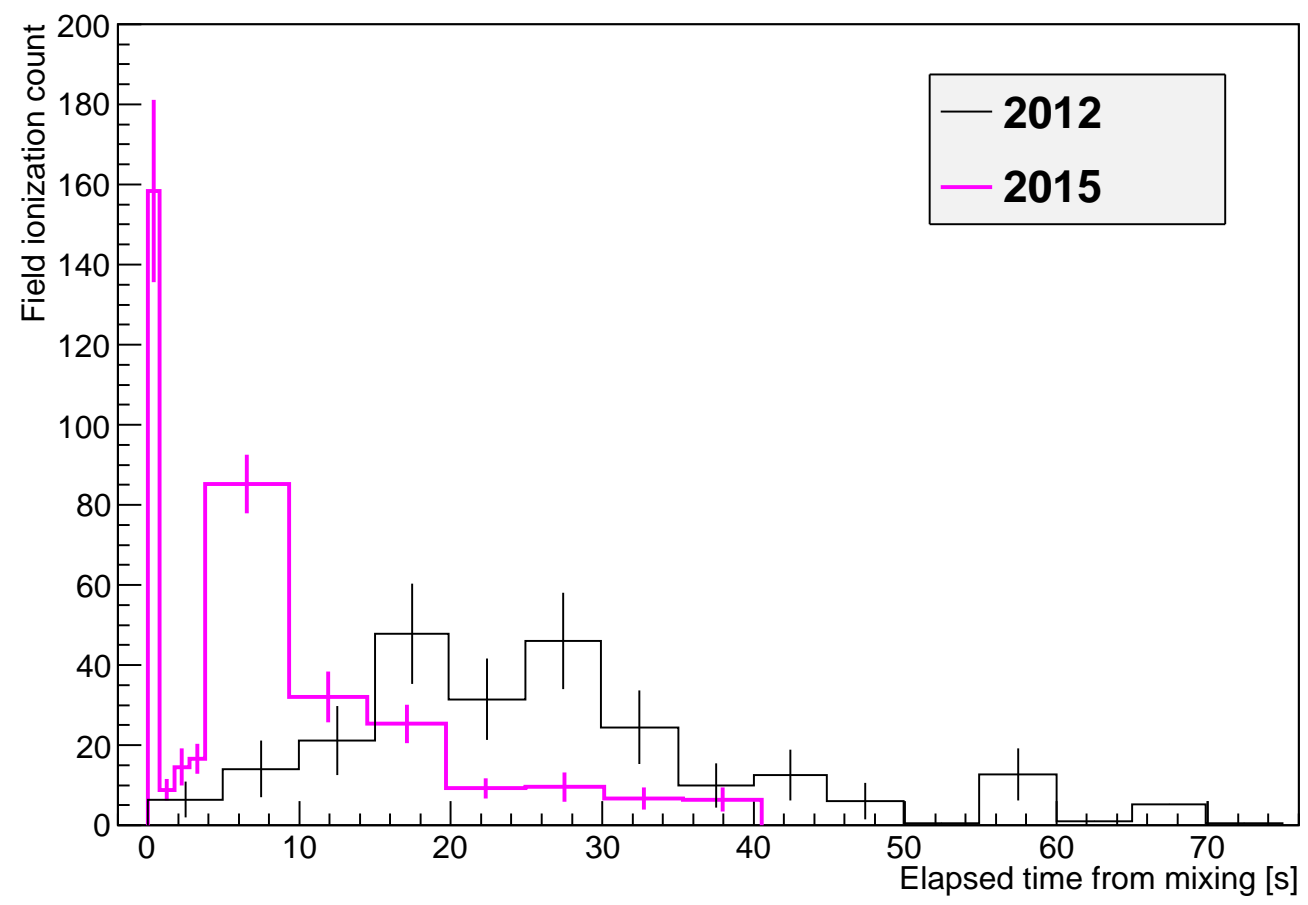

Fig. 6. Time dependences of antihydrogen production in 2015 (thick magenta line) and in 2012 (thin black line). For data in 2015, first 4 bins correspond to detected count for every $0.5 \mathrm{~s}$ and the others correspond to the count for every $5 \mathrm{~s}$.

It is noted that another peak is also seen at a few seconds, which means that some antiprotons are still energetic and so heat up positrons. Therefore the transport should be improved further so that antiprotons follow magnetic field lines more adiabatically. According to the calculation, the axial energy spread becomes smaller with increased current of the DC transport coils by a factor of 3 . It can be realized by a pulsed drive of the coils.

\section{Summary}

For an efficient production of antihydrogen atoms using the direct injection scheme, we developed the way to extract and transport antiprotons at $20 \mathrm{eV}$ more adiabatically than the previous scheme in 2012. Antihydrogen atoms were produced at higher rate immediately after the mixing, which implies the colder antihydrogen atoms. Further improvement will be realized by a modification of transport coils.

\section{References}

[1] N. Kuroda et al.: Phys. Rev. ST Accel. Beams 15 (2012) 024702.

[2] Y. Nagata and Y. Yamazaki.: New J. Phys. 16 (2014) 083026.

[3] N. Kuroda et al.: Nat. Commun. 5 (2014) 3089.

[4] N. Kuroda et al.: Phys. Rev. Lett. 100 (2008) 203402. 